\title{
11. Über die Gruppen, deren Darstellungen sich sämtlich auf monomiale Gestalt transformieren lassen.
}

\author{
By Kiyosi TAKETA. \\ Mathematical Institute, Tokyo Imperial University. \\ (Rec. Feb. 6, 1930. Comm. by T. Takagi, M.I.A., Feb. 12, 1930.)
}

Von den Gruppen, deren Darstellungen sämtlich als monomiale geschrieben werden können, sind die Sätze bekannt: ${ }^{\text {) }}$

(1) Die Kommutatorgruppe einer Gruppe von obiger Eigenschaft ist eine eigentliche Untergruppe oder $E$.

(2) Gruppen, deren Ordnung eine Primzahlpotenz oder ein Produkt von lauter von einander verschiedenen Primzahlen ist, läßt sich stets auf monomiale Gestalt transformieren.

Diese erweiternd wollen wir beweisen :

(A) Jede Gruppe $\mathbb{B}$ von der Eigenschaft des Titels ist auflösbar.

(B) Ist $\mathfrak{B}$ eine auflösbare Gruppe und $\mathfrak{A}$ ihr Abelscher Normalteiler der Art, daß die Hauptreihe der Faktorgruppe $\mathbb{S} / \mathfrak{A}$ zugleich die Kompositionsreihe wird, so lassen sich sämtliche Darstellungen von \&S auf monomiale Gestalt transformieren.

(I) Da unser Satz (A) für alle Abelschen Gruppen gilt, kann man vollständige Induktion anwenden. Es gibt immer holomorphe Darstellung von $\$ \$$, wie z. B. die reguläre Permutationsgruppe. Sei $\Gamma$ eine des niedrigsten Grades unter solchen. $\Gamma$ kann monomial angennomen werden, und ersetzen wir alle Einheitswurzeln bei ihr durch 1, dann erhalten wir eine Darstellung $\Gamma^{\prime}$ von $\$ 3$ durch Permutationen, die nicht mehr holomorph ist, weil sonst die Darstellung, die sich von $\Gamma^{\prime}$ durch die Wegnahme der identischen Darstellung ableiten lassen, gegen der Voraussetzung die holomorphe Darstellung von (\$) werden müßte. Also bilden diejenigen Elemente von $\Gamma$, welche dem Einheitselement von $\Gamma^{\prime}$ entsprechen einen Abelschen Normalteiler $\mathfrak{A}$ von $\mathbb{B}$. Da jede Darstellung von $\mathbb{B} / \mathfrak{A}$ aber diejenige von $\mathbb{B}$ wird, kann sie auf monomiale Gestalt transformieren, folglich muß $\mathfrak{B} / \mathfrak{A}$ auflösbar sein, denn sie ist von niedrigerer Ordnung als $\mathbb{S}$. Damit ist auch unser Satz bewiesen.

(II) Die Umkehrung von (A) ist nicht immer richtig. Z. B. die $139-140$

1) Vgl. A. Speiser, Theorie der Gruppen von endlicher Ordnung, Berlin (1923), 
Gruppe der Ordnung 24, (die durch $\left(\begin{array}{cc}-1 & 0 \\ 0 & -1\end{array}\right)$ amplificierte Tetraedergruppe) die durch die Matrizen

$$
T_{1}=\left(\begin{array}{cc}
i & 0 \\
0 & -i
\end{array}\right) \quad T_{2}=\left(\begin{array}{cc}
0 & -1 \\
1 & 0
\end{array}\right) \quad T_{3}=\left(\begin{array}{cc}
0 & i \\
i & 0
\end{array}\right) \quad \text { und } \quad S=\left(\begin{array}{cc}
\frac{-1+i}{2} & \frac{-1-i}{2} \\
\frac{1-i}{2} & \frac{-1-i}{2}
\end{array}\right)
$$

erzeugt wird," läßt sich nimmer als monomiale schreiben.

Beweis : Um die monomiale Gestalt zu haben, müßten die obigen drei Elemente $T$ entweder die Form

$$
\text { (1) }\left(\begin{array}{cc} 
\pm i & 0 \\
0 & \mp i
\end{array}\right) \text { oder (2) }\left(\begin{array}{cc}
0 & \omega \\
-\frac{1}{\omega} & 0
\end{array}\right)
$$

annehmen. Denn ihre Ordnung ist 4 und $|T|=1$. Da $S^{-1} T_{1} S=T_{2}$, $S^{-1} T_{2} S=T_{3}$, so enthält die Gruppe ein Element, das (1) in (2) transformiert. Dasselbe kann aber nicht monomial sein, denn aus der Beziehung

$$
\left(\begin{array}{ll}
a & b \\
c & d
\end{array}\right)\left(\begin{array}{cc} 
\pm i & 0 \\
0 & \mp i
\end{array}\right)=\left(\begin{array}{cc}
0 & \omega \\
-\frac{1}{\omega} & 0
\end{array}\right)\left(\begin{array}{ll}
a & b \\
c & d
\end{array}\right)
$$

ergibt sich die Gleichung $\pm a i=c \boldsymbol{c}$.

(III) Auch beim Beweise des Satzes (B) können wir die vollständige Induktion anwenden. Sei $\Gamma$ die gegebene Darstellung von $\$$. Es sind zwei Fälle, daß bei $\Gamma$ alle Matrizen von $\mathfrak{A}$ vollständig reduziert mindestens zwei verschiedene Darstellungen besitzen, oder nicht. Im ersten Falle ist (S) imprimitiv oder intransitiv.) Da es offenbar genügt den Satz für irreduzible Gruppen zu beweisen, so können wir annehmen, $\mathbb{B}$ sei imprimitiv, und erzeugt durch eine Darstellung einer Untergruppe $\mathfrak{S}^{2}$.) Die Frage wird also auf den Fall einer niedrigeren Ordnung zurückgeführt. Im zweiten Falle hat jede Matrix von $\mathfrak{A}$ Multiplikationsform, und $\mathbb{3}$ ein Zentrum 3. Da die Primfaktorgruppe der Hauptreihe einer Gruppe in ihrer Gesamtheit untereinander übereinstimmen, so muß es einen Normalteiler $\mathfrak{Q}$ von $(B)$ der Art geben, daß $\mathfrak{Q} / \mathbb{Z}$ eine Primzahlordnung hat. $\mathfrak{Q}$ ist offenbar Abelsch und bei $\Gamma$ enthält sie vollständig reduziert mindestens zwei verschiedene Darstellungen, denn sie hat noch andres Element als 3 .

1) Vgl. F. Klein, Vorlesungen über Ikosaeder, Leipzig (1884), 38.

2) Vgl. A. Speiser, l. c. 141. 
Der Satz ist also auf den ersten Fall zurückgeführt, da jede Primfaktorgruppe der Hauptreihe von $\mathfrak{S}$ eine Primzahlordnung hat, wie leicht einzusehen ist. Durch die Wiederholung dieser Schlußweise können wir unsre Behauptung beweisen, indem wir beachten, daß der Durchschnitt von $\mathfrak{S}$ und $\mathfrak{A}$ Abelscher Normalteiler von $\mathfrak{S}$ wird. 